\title{
EXISTENCE OF NON-TRIVIAL LIMIT CYCLES IN ABEL EQUATIONS WITH SYMMETRIES
}

\author{
M.J. ÁLVAREZ, J.L. BRAVO, AND M. FERNÁNDEZ
}

\begin{abstract}
We study the periodic solutions of the generalized Abel equation $x^{\prime}=a_{1} A_{1}(t) x^{n_{1}}+a_{2} A_{2}(t) x^{n_{2}}+a_{3} A_{3}(t) x^{n_{3}}$, where $n_{1}, n_{2}, n_{3}>$ 1 are distinct integers, $a_{1}, a_{2}, a_{3} \in \mathbb{R}$, and $A_{1}, A_{2}, A_{3}$ are $2 \pi$-periodic analytic functions such that $A_{1}(t) \sin t, A_{2}(t) \cos t, A_{3}(t) \sin t \cos t$ are $\pi$ periodic positive even functions.

When $\left(n_{3}-n_{1}\right)\left(n_{3}-n_{2}\right)<0$ we prove that the equation has no nontrivial (different from zero) limit cycle for any value of the parameters $a_{1}, a_{2}, a_{3}$.

When $\left(n_{3}-n_{1}\right)\left(n_{3}-n_{2}\right)>0$ we obtain under additional conditions the existence of non-trivial limit cycles. In particular, we obtain limit cycles not detected by Abelian integrals.
\end{abstract}

\section{Introduction and Main Result}

The number of limit cycles (periodic solutions isolated in the set of periodic solutions) of generalized Abel equations

$$
x^{\prime}=c_{1}(t) x+c_{2}(t) x^{2}+\ldots+c_{n}(t) x^{n},
$$

where $c_{1}, c_{2}, \ldots, c_{n}$ are $2 \pi$-periodic functions has been intensively studied due to its relation to Hilbert's 16th problem. This famous unsolved problem deals with the number and location of limit cycles of the planar system

$$
x^{\prime}=P(x, y), \quad y^{\prime}=Q(x, y),
$$

where $P(x, y), Q(x, y)$ are $n^{\text {th }}$-degree polynomials of $x$ and $y$.

When $P, Q$ are quadratic, this problem is equivalent to the determination of limit cycles of

$$
x^{\prime}=c_{1} x+c_{2}(t) x^{2}+c_{3}(t) x^{3},
$$

where $c_{1} \in \mathbb{R}$ and $c_{2}(t), c_{3}(t)$ are trigonometric polynomials [17]. Some higher degree planar systems, in particular rigid systems, can also be reduced to generalized Abel equations [9] [12, 13].

Even in the case $n=3$, the number of limit cycles of (1.1) is not bounded [17]. Thus, to obtain upper bounds for the number of limit cycles one must ask for some conditions to be set on the coefficients $c_{1}(t), c_{2}(t), \ldots, c_{n}(t)$.

2010 Mathematics Subject Classification. 34C25.

Key words and phrases. Periodic solutions; Abel equation.

M.J.A. was partially supported by grant MTM2008-03437. J.L.B. and M.F. were partially supported by grant FEDER(UE) MTM2008-05460. 
These additional conditions are that some of $c_{k}(t)$ have definite sign $[1,10$, $11,18]$, that $c_{n}(t) \equiv 1$ and the rest of the coefficients are bounded by a certain constant $[7,15]$, or that there exists a linear combination of some of the coefficients with definite sign $[4,5,14]$.

When (1.1) comes from a planar system, via Cherkas's transformation [8], the coefficients $c_{1}(t), \ldots, c_{n}(t)$ are trigonometric polynomials. Our aim is to explore the relation between limit cycles and the trigonometric monomials present in the coefficients.

To this end, let us consider the family of generalized Abel equations

$$
x^{\prime}=a_{1} A_{1}(t) x^{n_{1}}+a_{2} A_{2}(t) x^{n_{2}}+a_{3} A_{3}(t) x^{n_{3}}, \quad a_{1}, a_{2}, a_{3} \in \mathbb{R},
$$

where $n_{1}, n_{2}, n_{3} \geq 1$ are different fixed integers, and $A_{1}, A_{2}, A_{3}$ are $2 \pi$ periodic analytic functions. Note that $x(t) \equiv 0$ is always a periodic solution, which we shall call the trivial solution.

Throughout this communication, we shall assume the following conditions on the symmetries and the order of the functions $A_{k}$,

(A1) Assumptions on the symmetries of the functions: $A_{1}(t) \sin t, A_{2}(t) \cos t$, $A_{3}(t) \sin t \cos t$ are $\pi$-periodic positive even functions.

(A2) Assumptions on the order of the functions $A_{k}$ at the origin:

$$
A_{k}(t)=t^{i_{k}}+o\left(t^{i_{k}}\right) .
$$

(A3) Assumptions on the order of the functions $A_{k}$ at $\pi / 2$ :

$$
A_{k}\left(\frac{\pi}{2}-t\right)=\left(\frac{\pi}{2}-t\right)^{j_{k}}+o\left(\left(\frac{\pi}{2}-t\right)^{j_{k}}\right) .
$$

There is no restriction in assuming that the coefficients of $t^{i_{k}}$ and $\left(\frac{\pi}{2}-t\right)^{j_{k}}$ are 1 , because we shall obtain conditions that do not depend on the coefficients $a_{1}, a_{2}, a_{3}$.

The previous assumptions are satisfied for instance, when $A_{k}$ are trigonometric monomials, $A_{k}(t)=\sin ^{i_{k}} t \cos ^{j_{k}} t$, such that $i_{1}, j_{2}, i_{3}, j_{3}$ are odd and $j_{1}, i_{2}$ are even. The study of (1.2) in the case of $A_{k}$ being trigonometric monomials was begun in [2] and continued in [3]. In both papers, the existence of non-trivial limit cycles is characterized under certain hypotheses, in terms only of the symmetries of $A_{1}(t), A_{2}(t), A_{3}(t)$ and the number of different elements in the set $\left\{n_{1}, n_{2}, n_{3}\right\}$, except for the case studied in this present paper. The remaining case, i.e., the one with the symmetries of assumption (A1), presents a more complicated behaviour and can not be characterized just in terms of the symmetries and of the number of different $\left\{n_{k}\right\}$.

Let $u\left(t, x, a_{1}, a_{2}, a_{3}\right)$ denote the solution of (1.2) determined by the initial condition $u\left(0, x, a_{1}, a_{2}, a_{3}\right)=x$. To obtain periodic solutions, one only has to look for zeros of the displacement function defined as

$$
\Delta\left(x, a_{1}, a_{2}, a_{3}\right):=u\left(\pi, x, a_{1}, a_{2}, a_{3}\right)-u\left(-\pi, x, a_{1}, a_{2}, a_{3}\right) .
$$


Note that the sign of the displacement function is invariant under the election of the initial time, i.e., the sign of $u\left(t+2 \pi, x, a_{1}, a_{2}, a_{3}\right)-u\left(t, x, a_{1}, a_{2}, a_{3}\right)$ is invariant with respect to $t$.

Sometimes we shall simply write $\Delta(x)$ to denote $\Delta\left(x, a_{1}, a_{2}, a_{3}\right)$, assuming $a_{1}, a_{2}, a_{3}$ are fixed. For briefness, we shall denote the partial derivatives by a subindex and $u\left(t, x, a_{1}, a_{2}, a_{3}\right)$ as $u(t)$ when no confusion will arise.

Our focus will be on finding conditions on the exponents $n_{k}$ and on the order of the zero of $A_{k}$ at the origin or at $\pi / 2$ to determine whether there exist non-trivial limit cycles of Equation (1.2). Our main result is the following:

Theorem A. Consider Equation (1.2) where $A_{1}, A_{2}, A_{3}$ are $2 \pi$-periodic analytic functions such that $A_{1}(t) \sin t, A_{2}(t) \cos t, A_{3}(t) \sin t \cos t$ are $\pi$ periodic positive even functions. Assume that $A_{k}(t)=t^{i_{k}}+o\left(t^{i_{k}}\right)$ and $A_{k}(\pi / 2-t)=(\pi / 2-t)^{j_{k}}+o\left((\pi / 2-t)^{j_{k}}\right)$. Then the following statements hold:

(1) If $n_{k}=1$ for any $k \in\{1,2,3\}$, or $\left(n_{3}-n_{1}\right)\left(n_{3}-n_{2}\right)<0$, then (1.2) has no non-trivial limit cycle for any $a_{1}, a_{2}, a_{3} \in \mathbb{R}$.

(2) If $1<n_{3}<n_{2}<n_{1}$, and $\left(i_{1}+1\right)\left(n_{3}-1\right)>\left(i_{3}+1\right)\left(n_{1}-1\right)$, then there exist $a_{1}, a_{2}, a_{3}$ such that (1.2) has at least one non-trivial limit cycle.

(3) If $1<n_{3}<n_{1}<n_{2}$, and $\left(j_{2}+1\right)\left(n_{3}-1\right)>\left(j_{3}+1\right)\left(n_{2}-1\right)$, then there exist $a_{1}, a_{2}, a_{3}$ such that (1.2) has at least one non-trivial limit cycle.

The proof of the above theorem will be divided into two parts. In Section 2 we prove the first statement by analysing the sign of $u_{a_{2}}\left(\pi, x, a_{1}, a_{2}, a_{3}\right)$. Statements (2) and (3) are proved in Subsection 3.1 where we find positive zeros of $u_{a_{2}}\left(\pi, x, a_{1}, 0, a_{3}\right)$, analysing their behaviour near $x=0$ and $x=\infty$.

In Subsection 3.2, we study the bifurcation of the limit cycles by using Abelian integrals. The linear perturbations of the integrable centre $x^{\prime}=$ $a_{k} A_{k}(t) x^{n_{k}}$, i.e.,

$$
x^{\prime}=a_{k} A_{k}(t) x^{n_{k}}+\epsilon\left(a_{l} A_{l}(t) x^{n_{l}}+a_{m} A_{m}(t) x^{n_{m}}\right),
$$

where $1 \leq k, l, m \leq 3$ are distinct integers, do not give rise to any bifurcation of limit cycles, since $u_{\epsilon}\left(2 \pi, x, a_{1}, a_{2}, a_{3}\right)_{\mid \epsilon=0}$ is either identically null (when $k=3$ ) or does not change sign (when $k=1,2$ ). Consequently, we study the second-order perturbations, and then look for positive zeros of $u_{a_{1} a_{2}}\left(\pi, x, 0,0, a_{3}\right)$, analysing their behaviour near $x=0$ and $x=\infty$.

The present study is finally summarized in Theorem 3.3 in whose proof we obtain some results that complement the main one, showing that there exist limit cycles detected by Theorem A but not by Abelian integrals, and limit cycles detected by Abelian integrals but not by Theorem A. 


\section{Families with no Non-Trivial Limit Cycles}

In this section, we shall assume that $A_{1}, A_{2}, A_{3}$ are only $2 \pi$-periodic continuous functions.

Firstly, we solve the case when $n_{k}=1$ for any $k \in\{1,2,3\}$. If $n_{3}=1$, then the change of variable $x \rightarrow x \exp \left(a_{3} \int_{0}^{t} A_{3}(s) d s\right)$ preserves periodic solutions and transforms (1.2) with the assumed symmetries (A1) into

$$
x^{\prime}=f_{1}(t) x^{n_{1}}+f_{2}(t) x^{n_{2}},
$$

where $f_{1}(t)$ is odd and does not change sign in $(0, \pi)$, and $f_{2}(t-\pi / 2)$ is odd and does not change sign in $(\pi / 2,3 \pi / 2)$. From [6, Theorem 24$]$, one obtains that (1.2) has no non-trivial limit cycle. When $n_{k}=1, k=1,2$, the change of variable $x \rightarrow x \exp \left(a_{k} \int_{0}^{t} A_{k}(s) d s\right)$ transforms (1.2) with the assumed symmetries into

$$
x^{\prime}=f_{j}(t) x^{n_{j}}+f_{3}(t) x^{n_{3}}, \quad j \in\{1,2\}, j \neq k,
$$

where $f_{j}(t)$ and $f_{3}(t)$ are odd. Then every bounded solution is periodic. Since $\Delta(x)$ is analytic, there is no non-trivial limit cycle. Henceforth, therefore, we shall assume that $n_{k}>1, k=1,2,3$.

The first comment is that the change $x \rightarrow-x$ does not change the family (just the signs of some of the coefficients $a_{1}, a_{2}, a_{3}$ ). Henceforth, therefore, we shall only study the existence or non-existence of positive limit cycles.

To prove the existence of positive limit cycles when $\left(n_{3}-n_{1}\right)\left(n_{3}-n_{2}\right)<0$, we shall first prove that if $a_{1}, a_{3}$ are fixed then $a_{2} \rightarrow \Delta\left(x, a_{1}, a_{2}, a_{3}\right)$ is monotonic (its derivative has constant sign), and using that for $a_{2}=0$ every bounded solution is periodic (one has a centre), we conclude.

Proposition 2.1. Assume that $A_{1}, A_{2}, A_{3}$ are $2 \pi$-periodic continuous functions such that $A_{1}(t) \sin t, A_{2}(t) \cos t, A_{3}(t) \sin t \cos t$ are $\pi$-periodic positive even functions. Given $x, a_{1}, a_{2}, a_{3}$ such that $u\left(t, x, a_{1}, a_{2}, a_{3}\right)$ is defined in $[-\pi, \pi]$, one has

$$
u_{a_{2}}(\pi)=u^{n_{2}}(\pi) \int_{0}^{\pi / 2} A_{2}(t)\left(e^{\int_{t}^{\pi} L_{2}(s) d s}-e^{\int_{\pi-t}^{\pi} L_{2}(s) d s}\right) d t,
$$

where

$$
L_{2}(s)=\left(n_{1}-n_{2}\right) a_{1} A_{1}(s) u^{n_{1}-1}(s)+\left(n_{3}-n_{2}\right) a_{3} A_{3}(s) u^{n_{3}-1}(s) .
$$

Moreover, if $u\left(t, x, a_{1}, \bar{a}_{2}, a_{3}\right)$ is defined in $[-\pi, \pi]$ for every $\left|\bar{a}_{2}\right| \leq\left|a_{2}\right|$, then there exists $\bar{a}_{2}^{*} \in\left[-a_{2}, a_{2}\right]$ such that

$$
u\left(\pi, x, a_{1}, a_{2}, a_{3}\right)-u\left(-\pi, x, a_{1}, a_{2}, a_{3}\right)=2 u_{a_{2}}\left(\pi, x, a_{1}, \bar{a}_{2}^{*}, a_{3}\right) a_{2} .
$$

Proof. By symmetries,

$$
u\left(-t, x, a_{1}, a_{2}, a_{3}\right)=u\left(t, x, a_{1},-a_{2}, a_{3}\right) .
$$

Now (2.4) follows from the Mean Value Theorem. 
Firstly, differentiating (1.2) with respect to $a_{2}$,

$$
u_{a_{2}}^{\prime}=A_{2} u^{n_{2}}+\left(a_{1} n_{1} A_{1} u^{n_{1}-1}+a_{2} n_{2} A_{2} u^{n_{2}-1}+a_{3} n_{3} A_{3} u^{n_{3}-1}\right) u_{a_{2}}
$$

Since $u^{\prime}=a_{1} A_{1} u^{n_{1}}+a_{2} A_{2} u^{n_{2}}+a_{3} A_{3} u^{n_{3}}$, then

$$
a_{2} A_{2} u^{n_{2}-1}=\frac{u^{\prime}}{u}-a_{1} A_{1} u^{n_{1}-1}-a_{3} A_{3} u^{n_{3}-1} .
$$

Substituting the above equality into (2.5), and integrating from 0 to $\pi$, one has

$$
\begin{aligned}
u_{a_{2}}(\pi) & =\int_{0}^{\pi} A_{2}(t) u^{n_{2}}(t) e^{\int_{t}^{\pi} n_{2} \frac{u^{\prime}}{u}+a_{1}\left(n_{1}-n_{2}\right) A_{1} u^{n_{1}-1}+a_{3}\left(n_{3}-n_{2}\right) A_{3} u^{n_{3}-1} d s} d t \\
& =u^{n_{2}}(\pi) \int_{0}^{\pi} A_{2}(t) e^{\int_{t}^{\pi} a_{1}\left(n_{1}-n_{2}\right) A_{1} u^{n_{1}-1}+a_{3}\left(n_{3}-n_{2}\right) A_{3} u^{n_{3}-1} d s} d t .
\end{aligned}
$$

Now, taking into account that $A_{2}(\pi / 2-t)$ is odd, we conclude (2.3).

Proposition 2.2. Assume that $A_{1}, A_{2}, A_{3}$ are $2 \pi$-periodic continuous functions such that $A_{1}(t) \sin t, A_{2}(t) \cos t, A_{3}(t) \sin t \cos t$ are $\pi$-periodic positive even functions, and let $u(t)>0$ be a solution of (1.2) defined for every $t \in[0, \pi]$. Then

$$
\operatorname{sign}(u(t)-u(\pi-t))=\operatorname{sign}\left(-a_{1}\right), \quad \text { for every } t \in(0, \pi / 2) .
$$

Proof. Assume there exists $t_{0}$ such that $u\left(t_{0}\right)=u\left(\pi-t_{0}\right)$. Then, by symmetries,

$$
(u(t)-u(\pi-t))^{\prime}=2 a_{1} A_{1}(t) u^{n_{1}}(t) .
$$

Since for $t=\pi / 2, u(t)=u(\pi-t)$, the claim holds.

We recall that a function $\alpha(t)$ is an upper solution (respectively lower solution) of the equation $x^{\prime}=f(t, x)$ provided that $\alpha^{\prime}(t) \geq f(t, \alpha(t))$ (resp. $\left.\alpha^{\prime}(t) \leq f(t, \alpha(t))\right)$.

We can now restate the first part of Theorem A, and prove it.

Theorem 2.3. Assume that $A_{1}, A_{2}, A_{3}$ are $2 \pi$-periodic continuous functions such that $A_{1}(t) \sin t, A_{2}(t) \cos t, A_{3}(t) \sin t \cos t$ are $\pi$-periodic positive even functions, and $\left(n_{1}-n_{3}\right)\left(n_{2}-n_{3}\right)<0$. Then there is no non-trivial limit cycle of (1.2) for any value of $a_{1}, a_{2}, a_{3} \in \mathbb{R}$.

Proof. Assume the following:

Claim. Fix $x, a_{1}, a_{2}, a_{3}$ such that $u\left(\pi, x, a_{1}, a_{2}, a_{3}\right)$ is defined. Then $u\left(\pi, x, a_{1}, \bar{a}_{2}, a_{3}\right)$ is defined for each $\left|\bar{a}_{2}\right|<\left|a_{2}\right|$.

As a consequence one has that $u_{a_{2}}\left(\pi, x, a_{1}, \bar{a}_{2}, a_{3}\right)$ is also defined for every $\left|\bar{a}_{2}\right|<a_{2}$, and Proposition 2.1 holds.

Given $a_{1}, a_{3}$ we shall prove that $\operatorname{sign}\left(n_{1}-n_{2}\right)=\operatorname{sign}\left(a_{3}\left(n_{2}-n_{3}\right)\right)$ implies that $u_{a_{2}}\left(\pi, x, a_{1}, a_{2}, a_{3}\right)$ has definite sign for every $x, a_{2}$ whenever it is defined. As a consequence of (2.4), we shall obtain that Equation (1.2) has no non-trivial limit cycles. Applying the above arguments to (1.2) after the change of variable $t \rightarrow \pi / 2-t$, we shall prove that $\operatorname{sign}\left(n_{1}-n_{2}\right)=$ $\operatorname{sign}\left(a_{3}\left(n_{1}-n_{3}\right)\right)$ implies that Equation (1.2) has no non-trivial limit cycles. 
Finally we shall show that if $\left(n_{1}-n_{3}\right)\left(n_{2}-n_{3}\right)<0$ then Equation (1.2) has no non-trivial limit cycles for any $a_{1}, a_{2}, a_{3}$.

By $(2.3)$, if

$$
e^{\int_{t}^{\pi} L_{2}(s) d s}-e^{\int_{\pi-t}^{\pi} L_{2}(s) d s}, \quad t \in(0, \pi / 2),
$$

has definite sign, where

$$
L_{2}(s)=\left(n_{1}-n_{2}\right) a_{1} A_{1}(s) u^{n_{1}-1}(s)+\left(n_{3}-n_{2}\right) a_{3} A_{3}(s) u^{n_{3}-1}(s),
$$

then $u_{a_{2}}(\pi)$ has the same sign. Therefore it suffices to show that

$$
\int_{t}^{\pi-t} L_{2}(s) d s, \quad t \in(0, \pi / 2)
$$

has definite sign. This last expression can be rewritten, taking into account the symmetries of $A_{1}$ and $A_{3}$, as

$$
\begin{aligned}
& \int_{t}^{\pi / 2}\left(n_{1}-n_{2}\right) a_{1} A_{1}(s)\left(u^{n_{1}-1}(s)+u^{n_{1}-1}(\pi-s)\right) \\
& \quad+\left(n_{3}-n_{2}\right) a_{3} A_{3}(s)\left(u^{n_{3}-1}(s)-u^{n_{3}-1}(\pi-s)\right) d s, \quad t \in(0, \pi / 2) .
\end{aligned}
$$

The above expression has definite sign when its integrand has definite sign.

By Proposition 2.2,

$$
\operatorname{sign}\left(u^{n_{1}-1}(s)-u^{n_{1}-1}(\pi-s)\right)=\operatorname{sign}\left(-a_{1}\right) .
$$

Thus, if

$$
\operatorname{sign}\left(n_{1}-n_{2}\right)=\operatorname{sign}\left(a_{3}\left(n_{2}-n_{3}\right)\right),
$$

then $u_{a_{2}}(\pi)$ has definite sign and Equation (1.2) has no non-trivial limit cycles.

By the change of variable $t \rightarrow \pi / 2-t$, (1.2) becomes

$$
x^{\prime}=-a_{1} A_{1}(\pi / 2-t) x^{n_{1}}-a_{2} A_{2}(\pi / 2-t) x^{n_{2}}-a_{3} A_{3}(\pi / 2-t) x^{n_{3}},
$$

where now the symmetries of the functions $A_{1}$ and $A_{2}$ have been interchanged, i.e., $A_{1}(\pi / 2-t) \cos t, A_{2}(\pi / 2-t) \sin t$, are $\pi$-periodic positive even functions. Repeating the above arguments, one obtains that if

$$
\operatorname{sign}\left(n_{2}-n_{1}\right)=\operatorname{sign}\left(-a_{3}\left(n_{1}-n_{3}\right)\right)
$$

then Equation (2.6) has no non-trivial limit cycles. Therefore, neither does (1.2).

By hypothesis, $\operatorname{sign}\left(n_{1}-n_{3}\right)=-\operatorname{sign}\left(n_{2}-n_{3}\right)$. Then, for each $a_{3}$, either $\operatorname{sign}\left(n_{1}-n_{2}\right)=\operatorname{sign}\left(a_{3}\left(n_{2}-n_{3}\right)\right)$ or $\operatorname{sign}\left(n_{1}-n_{2}\right)=\operatorname{sign}\left(a_{3}\left(n_{1}-n_{3}\right)\right)$. In both cases we conclude that Equation (1.2) has no non-trivial limit cycles.

Proof of the Claim. Under our hypotheses

$$
\operatorname{sign} u_{a_{2}}(\pi)=\operatorname{sign} L_{2}(s)=\operatorname{sign}\left(\left(n_{1}-n_{2}\right) a_{1}\right) .
$$

Thus, by the change of variable $t \rightarrow \pi+t$, one may choose the sign of $a_{1}$ such that $u_{a_{2}}\left(\pi, x, a_{1}, a_{2}, a_{3}\right)>0$. Note that there is no restriction of assuming $a_{2}>0$. 
Define $u(t, x, \lambda)=u\left(t, x, a_{1}, \lambda a_{2}, a_{3}\right)$. Since $a_{2}>0$, one has that $u(t, x, 1)$ is an upper solution of

$$
x^{\prime}=a_{1} A_{1}(t) x^{n_{1}}+\lambda a_{2} A_{2}(t) x^{n_{2}}+a_{3} A_{3}(t) x^{n_{3}}
$$

for every $\lambda<1, t \in[0, \pi / 2]$, and a lower solution for every $\lambda<1, t \in[\pi / 2, \pi]$. Writing

$$
I=\left\{\lambda_{0} \in(-1,1): u(t, x, \lambda)<u(t, x, 1), t \in(0, \pi], \lambda_{0} \leq \lambda<1\right\},
$$

we shall prove that $I$ is a non-empty, open and closed subset of $(-1,1)$, i.e., $I=(-1,1)$. Note that $u_{\lambda}(\pi, x, \lambda)=a_{2} u_{a_{2}}\left(\pi, x, a_{1}, \lambda a_{2}, a_{3}\right)>0$. Then, for any $\lambda_{0}<1$ close enough to $1, u\left(\pi, x, \lambda_{0}\right)<u(\pi, x, 1)$. Since $u\left(0, x, \lambda_{0}\right)=$ $u(0, x, 1)=x$ and $u(t, x, 1)$ is an upper-solution for $t \in[0, \pi / 2]$, then $u\left(t, x, \lambda_{0}\right)<u(t, x, 1)$ for every $t \in(0, \pi / 2]$, and since $u\left(\pi, x, \lambda_{0}\right)<u(\pi, x, 1)$ and $u(t, x, 1)$ is a lower-solution for $t \in[\pi / 2, \pi]$, then $u\left(t, x, \lambda_{0}\right)<u(t, x, 1)$ for every $t \in[\pi / 2, \pi)$. Therefore $I$ is not empty. Let $\lambda_{0} \in I$. By definition of $I, u(\pi, x, \lambda)<u(\pi, x, 1)$ for $\lambda_{0} \leq \lambda<1$, and the same inequality holds for close enough $\lambda<\lambda_{0}$ since $u_{\lambda}(\pi, x, \lambda)>0$. Thus, the above arguments show that $I$ is open. To prove that $I$ is a closed subset of $(-1,1)$, let $\lambda_{n} \rightarrow \lambda_{0} \in(-1,1)$ and $\lambda_{n} \in I$. Then, for each $t \in[0, \pi]$ belonging to the interval of the definition of $u\left(t, x, \lambda_{0}\right)$, one has

$$
u\left(t, x, \lambda_{n}\right) \rightarrow u\left(t, x, \lambda_{0}\right), \quad u\left(t, x, \lambda_{0}\right) \leq u(t, x, 1) .
$$

As a consequence, one has that $u\left(\pi, x, \lambda_{0}\right)$ is defined. Necessarily $\lambda_{n}>\lambda_{0}$, since otherwise $\lambda_{0} \in I$. Since $u_{\lambda}\left(\pi, x, \lambda_{0}\right)>0$, if $u\left(\pi, x, \lambda_{0}\right)=u(\pi, x, 1)$ then

$$
u\left(\pi, x, \lambda_{n}\right)>u\left(\pi, x, \lambda_{0}\right)=u(\pi, x, 1)
$$

for $\lambda_{n}$ sufficiently close to $\lambda_{0}$, in contradiction with $\lambda_{n} \in I$. Therefore, $u\left(\pi, x, \lambda_{0}\right)<u(\pi, x, 1)$, and repeating the arguments above, we get $\lambda_{0} \in$ $I$.

\section{Families with At Least One Non-Trivial Limit Cycle}

In this section we shall present two criteria for the existence of non-trivial limit cycles. These criteria will be obtained by two different methods. In Subsection 3.1, we study the order of the solution at infinity. We will thereby be able to prove the remaining statements of the main theorem. In Subsection 3.2, as was mentioned in the Introduction, we study the bifurcation of limit cycles by the linear perturbation of an integrable centre. As the first-order perturbations do not give rise to any limit cycle, we study the second-order ones. Applying this method, we obtain some additional results that give conditions different from those in the main theorem.

3.1. Proof of Theorem A. In the following we prove a preliminary result studying the order of the solution at infinity and its behaviour.

Proposition 3.1. Assume that $A_{1}, A_{2}, A_{3}$ are $2 \pi$-periodic analytic functions such that $A_{1}(t) \sin t, A_{2}(t) \cos t, A_{3}(t) \sin t \cos t$ are $\pi$-periodic positive 
even functions, $n_{3}<n_{2}<n_{1}, a_{2}=0$, and $a_{1}, a_{3}<0$ are fixed. Denote by $i_{k}$ the order of $A_{k}$ at the origin, and

$$
u(t, \infty)=\sup \left\{u\left(t, x, a_{1}, a_{2}, a_{3}\right): x>0\right\} .
$$

Then

(1) Every bounded solution of (1.2) is periodic. Moreover, $u(t, x)$ is defined on $[0,2 \pi]$ for every $x>0$, and $u(t, x)>u(\pi-t, x)$ for every $t \in(0, \pi / 2)$.

(2) The function $u(t, \infty)$ is a solution of (1.2) defined for $t \in(0,2 \pi)$, $u(t, \infty) \rightarrow+\infty$ as $t \rightarrow 0$, and

$$
u(t, \infty)=\left\{\begin{array}{lll}
\left(\left(1-n_{1}\right) a_{3}\right)^{\frac{1}{1-n_{1}}} t^{\frac{i_{3}+1}{1-n_{3}}}+o\left(t^{\frac{i_{3}+1}{1-n_{3}}}\right), & \text { if } \frac{i_{1}+1}{1-n_{1}}<\frac{i_{3}+1}{1-n_{3}}, \\
\left(\left(1-n_{1}\right) a_{1}\right)^{\frac{1}{1-n_{1}}} t^{\frac{i_{1}+1}{1-n_{1}}}+o\left(t^{\frac{i_{1}+1}{1-n_{1}}}\right), & \text { if } \frac{i_{1}+1}{1-n_{1}}>\frac{i_{3}+1}{1-n_{3}} .
\end{array}\right.
$$

(3) If $\left(i_{1}+1\right)\left(n_{3}-1\right)>\left(i_{3}+1\right)\left(n_{1}-1\right)$, then for $a_{3}<0$ small enough

$$
u_{a_{2}}\left(\pi, x, a_{1}, 0, a_{3}\right)>0 \text { for } x \text { sufficiently large. }
$$

Proof. (1) Since $a_{2}=0$, one has that the equation is invariant under the change of variable $t \rightarrow-t$. Therefore, the solutions are even and hence $2 \pi$ periodic $(u(-\pi, x)=u(\pi, x)$ whenever it is defined).

Let us show that $u(t, x)$ is defined in $[0,2 \pi]$ (and hence periodic). It suffices to prove that $u(t, x)$ is defined in $[0, \pi]$, because $u(2 \pi-t, x)$ is also a solution. Since $a_{1}, a_{3}<0$, then $u(t, x)$ is decreasing for $t \in[0, \pi / 2]$, and consequently is defined. Finally, $u(\pi-t, x)$ is an upper-solution in $[\pi / 2, \pi]$, so that $u(t, x)$ is defined in $[\pi / 2, \pi]$. Moreover, $u(\pi-t, x)<u(t, x)$ for every $t \in(0, \pi / 2)$.

(2) Since the solutions are even, decreasing in $[0, \pi / 2]$ and $u(\pi-t, x)$ is an upper solution, it suffices to prove that $u(t, \infty)$ is defined in $(0, \pi)$.

By the change of variables $y=x^{1-n_{1}}$, one obtains that any positive solution of (1.2) corresponds to a positive solution of

$$
y^{\prime}=\left(1-n_{1}\right) a_{3} A_{3} y^{\alpha}+\left(1-n_{1}\right) a_{1} A_{1} .
$$

where $0<\alpha=\frac{n_{3}-n_{1}}{1-n_{1}}<1$. Let $v(t)$ be the solution of (3.9) such that $v(0)=0$ (the uniqueness follows from [16]). Since (3.9) has no positive solution with initial condition at $(\pi, 0)$, then $v(\pi)>0$. Hence

$$
u(t, x)=v\left(t, x^{1-n_{1}}\right)^{\frac{1}{1-n_{1}}}, \quad u(t, \infty)=v(t, 0)^{\frac{1}{1-n_{1}}},
$$

for every $t \in(0, \pi]$.

But

$$
v(t, 0)=\left(1-n_{1}\right) a_{3} \int_{0}^{t} A_{3}(s) v(s, 0)^{\alpha} d s+\left(1-n_{1}\right) a_{1} \int_{0}^{t} A_{1}(s) d s .
$$

Hence,

$$
v(t, 0)=\left(1-n_{1}\right) a_{3} t^{\left(i_{3}+1\right) /(1-\alpha)} O(1) \quad \text { or } \quad v(t, 0)=\left(1-n_{1}\right) a_{1} t^{i_{1}+1} O(1) .
$$

Therefore, (3.8) holds. 
(3) As in Proposition 2.1, one has

$$
u_{a_{2}}(\pi)=u^{n_{1}}(\pi) \int_{0}^{\pi} A_{2}(t) e^{\int_{t}^{\pi} L_{2}(s, x) d s} d t
$$

where

$$
L_{2}(t, x)=\left(n_{1}-n_{2}\right) a_{1} A_{1}(t) u^{n_{1}-1}(t, x)+\left(n_{3}-n_{2}\right) a_{3} A_{3}(t) u^{n_{3}-1}(t, x) .
$$

By hypothesis, $\left(i_{1}+1\right) /\left(1-n_{1}\right)<\left(i_{3}+1\right) /\left(1-n_{3}\right)$, so that

$$
u(t, \infty)=\left(\left(1-n_{1}\right) a_{3}\right)^{\frac{1}{1-n_{1}}} t^{\frac{1+i_{3}}{1-n_{3}}}+o\left(t^{\frac{1+i_{3}}{1-n_{3}}}\right) .
$$

In consequence, the first summand of $L_{2}(t, x)$ is

$$
\left(n_{1}-n_{2}\right) a_{1} A_{1}(s) u^{n_{1}-1}(s, \infty)=\frac{\left(n_{1}-n_{2}\right) a_{1}}{\left(1-n_{1}\right) a_{3}} s^{i_{1}+\frac{\left(1+i_{3}\right)\left(n_{1}-1\right)}{\left(1-n_{3}\right)}}+o\left(s^{i_{1}+\frac{\left(1+i_{3}\right)\left(n_{1}-1\right)}{\left(1-n_{3}\right)}}\right),
$$

where

$$
i_{1}+\frac{\left(1+i_{3}\right)\left(n_{1}-1\right)}{\left(1-n_{3}\right)}>-1
$$

and the second summand of $L_{2}(t, x)$ is

$$
\left(n_{3}-n_{2}\right) a_{3} A_{3}(s) u^{n_{3}-1}(s, \infty)=\left(n_{3}-n_{2}\right) a_{3}\left(\left(1-n_{1}\right) a_{3}\right)^{\frac{n_{3}-1}{1-n_{1}}} s^{-1}+o\left(s^{-1}\right) .
$$

Then

$$
\begin{aligned}
\exp \left(\int_{t}^{\pi} L_{2}(s, \infty) d s\right) & =\exp \left(\left(n_{3}-n_{2}\right) a_{3}\left(\left(1-n_{1}\right) a_{3}\right)^{\frac{n_{3}-1}{1-n_{1}}} \int_{t}^{\pi}\left(s^{-1}+o\left(s^{-1}\right)\right) d s\right) \\
& =K t^{\left(n_{2}-n_{3}\right) a_{3}\left(\left(1-n_{1}\right) a_{3}\right)^{\frac{n_{3}-1}{1-n_{1}}}}+o\left(t^{\left(n_{2}-n_{3}\right) a_{3}\left(\left(1-n_{1}\right) a_{3}\right)^{\frac{n_{3}-1}{1-n_{1}}}}\right),
\end{aligned}
$$

where

$$
K=\pi^{\left(n_{3}-n_{2}\right) a_{3}\left(\left(1-n_{1}\right) a_{3}\right)^{\frac{n_{3}-1}{1-n_{1}}}>0 .}
$$

Since $0<1+\left(n_{3}-1\right) /\left(1-n_{1}\right)<1$, one can choose $a_{3}<0$ such that

$$
1+i_{2}+\left(n_{2}-n_{3}\right) a_{3}\left(\left(1-n_{1}\right) a_{3}\right)^{\frac{n_{3}-1}{1-n_{1}}}<0 .
$$

Therefore,

$$
\int_{0}^{\pi} A_{2}(t) \exp \left(\int_{t}^{\pi} L_{2}(s, \infty) d s\right) d t=\infty .
$$

To end the proof, we need to show that

$$
\int_{0}^{\pi} A_{2}(t) \exp \left(\int_{t}^{\pi} L_{2}(s, x) d s\right) d t \rightarrow \int_{0}^{\pi} A_{2}(t) \exp \left(\int_{t}^{\pi} L_{2}(s, \infty) d s\right) d t=\infty
$$

as $x \rightarrow \infty$. Indeed, by the Dominated Convergence Theorem

$$
\int_{\pi / 2}^{\pi} A_{2}(t) \exp \left(\int_{t}^{\pi} L_{2}(s, x) d s\right) d t \rightarrow \int_{\pi / 2}^{\pi} A_{2}(t) \exp \left(\int_{t}^{\pi} L_{2}(s, \infty) d s\right) d t
$$


as $x \rightarrow \infty$. Since

$$
\begin{gathered}
L_{2}(t, x) \rightarrow L_{2}(t, \infty), \text { as } x \rightarrow \infty, \\
\exp \left(\int_{\pi / 2}^{\pi} L_{2}(t, \infty) d t\right)<\infty,
\end{gathered}
$$

the Dominated Convergence Theorem guarantees that

$$
\exp \left(\int_{\pi / 2}^{\pi} L_{2}(t, x) d t\right) \rightarrow \exp \left(\int_{\pi / 2}^{\pi} L_{2}(t, \infty) d t\right) \text { as } x \rightarrow \infty .
$$

Let $L_{2}(t, x)=L_{21}(t, x)+L_{22}(t, x)$, where

$$
\begin{aligned}
& L_{21}(t, x)=\left(n_{1}-n_{2}\right) a_{1} A_{1}(t) u^{n_{1}-1}(t, x), \\
& L_{22}(t, x)=\left(n_{3}-n_{2}\right) a_{3} A_{3}(t) u^{n_{3}-1}(t, x) .
\end{aligned}
$$

For $t \in(0, \pi / 2], L_{2 k}(t, x) \rightarrow L_{2 k}(t, \infty)$ as $x \rightarrow \infty$, the convergence being monotonic decreasing for $k=1$ and monotonic increasing for $k=2$. So,

$$
\exp \left(\int_{t}^{\pi / 2} L_{2 k}(s, x) d s\right) \rightarrow \exp \left(\int_{t}^{\pi / 2} L_{2 k}(s, \infty) d s\right)
$$

as $x \rightarrow \infty$, the convergence being monotonic decreasing for $k=1$ and monotonic increasing for $k=2$. Then

$$
\begin{aligned}
& \int_{0}^{\pi / 2} A_{2}(t) \exp \left(\int_{t}^{\pi / 2}\left(L_{21}(s, x)+L_{22}(s, x)\right) d s\right)> \\
& \int_{0}^{\pi / 2} A_{2}(t) \exp \left(\int_{t}^{\pi / 2} L_{21}(s, \infty) d s\right) \exp \left(\int_{t}^{\pi / 2} L_{22}(s, x) d s\right) d t \\
& \rightarrow \int_{0}^{\pi / 2} A_{2}(t) \exp \left(\int_{t}^{\pi / 2} L_{2}(s, \infty) d s\right) d t=\infty .
\end{aligned}
$$

Now we can restate and prove the last parts of Theorem A.

Theorem 3.2. Assume that $A_{1}, A_{2}, A_{3}$ are $2 \pi$-periodic analytic functions such that $A_{1}(t) \sin t, A_{2}(t) \cos t, A_{3}(t) \sin t \cos t$ are $\pi$-periodic positive even functions, and denote by $i_{k}, j_{k}$ the orders of $A_{k}$ at the origin and at $\pi / 2$, respectively.

If $n_{3}<n_{2}<n_{1}$, and $\left(i_{1}+1\right)\left(n_{3}-1\right)>\left(i_{3}+1\right)\left(n_{1}-1\right)$, then there exist $a_{1}, a_{2}, a_{3}$ such that (1.2) has one non-trivial limit cycle.

The same holds if $n_{3}<n_{1}<n_{2}$ and $\left(j_{2}+1\right)\left(n_{3}-1\right)>\left(j_{3}+1\right)\left(n_{2}-1\right)$.

Proof. Let $a_{1}, a_{3}<0$. By Proposition 3.1, the stability for $x$ sufficiently large and $a_{2}$ small is determined by the sign of $a_{2}$. The stability at the origin is 
determined by the sign of $a_{2}\left(n_{2}-n_{1}\right)$ because

$$
\begin{aligned}
\operatorname{sign} \Delta\left(x, a_{1}, a_{2}, a_{3}\right) & =\operatorname{sign}\left(u\left(2 \pi, x, a_{1}, a_{2}, a_{3}\right)-x\right) \\
& =\operatorname{sign}\left(a_{1} a_{2}\left(n_{2}-n_{1}\right) I_{A_{2} A_{1}}(2 \pi)+o\left(a_{1}^{2}, a_{1} a_{2}, a_{2}^{2}\right)\right),
\end{aligned}
$$

where

$$
I_{A_{2} A_{1}}(2 \pi)=\int_{0}^{2 \pi} A_{2}(t)\left(\int_{0}^{t} A_{1}(s) d s\right) d t<0 .
$$

See [2, Appendix A, (A34)-(A39)].

Since the above two stability-determining signs are opposite, there is one non-trivial limit cycle for $a_{1}, a_{3}<0<a_{2}$ and $a_{2}$ small enough.

To prove the second part of the result it is enough to perform the change of variable $t \rightarrow \pi / 2-t$ and apply the statement already proved.

3.2. Second-order perturbation of an integrable centre. In this subsection we shall obtain some criteria for the existence of non-trivial limit cycles by Abelian integrals. As we noted in the Introduction, the linear perturbations of the integrable centre given by $x^{\prime}=a_{k} A_{k}(t) x^{n_{k}}$ do not give rise to the detection of limit cycles. We therefore study the second-order perturbations, obtained by perturbing by $a_{1}, a_{2}$ the centre given by $a_{1}=a_{2}=0$.

Theorem 3.3. Assume that $A_{1}, A_{2}, A_{3}$ are $2 \pi$-periodic analytic functions such that $A_{1}(t) \sin t, A_{2}(t) \cos t, A_{3}(t) \sin t \cos t$ are $\pi$-periodic positive even functions. and denote by $i_{k}, j_{k}$ the orders of $A_{k}$ at the origin and at $\pi / 2$, respectively. Moreover, assume that one of the following conditions holds:

$\left(C_{1}\right) \quad(a) n_{1}, n_{2}<n_{3}$,

(b) $\frac{i_{2}+1}{i_{3}+1} \leq \frac{n_{2}-1}{n_{3}-1}$

(c) $\left(n_{1}-n_{2}\right)\left(\frac{n_{3}}{k_{2}}-n_{2}\right)<0$.

$\left(C_{2}\right) \quad(a) n_{3}<n_{1}, n_{2}, \quad \frac{i_{1}+1}{i_{3}+1}<\frac{n_{1}-n_{3}}{n_{3}-1}, \quad \frac{i_{2}+1}{i_{3}+1}>\frac{n_{2}-n_{3}}{n_{3}-1}$,

(b) $\frac{i_{1}+1+i_{2}+1}{i_{3}+1} \leq \frac{n_{1}+n_{2}-n_{3}-1}{n_{3}-1}$,

(c) $\left(n_{1}-n_{2}\right)\left(\frac{n_{1}}{k_{2}}-\frac{n_{2}}{k_{1}}+\frac{n_{3}}{k_{1} k_{2}}\right)<0$,

where

$$
k_{l}=1+i_{l}-\left(n_{l}-n_{3}\right) \frac{i_{3}+1}{n_{3}-1}, \quad l=1,2 .
$$

Then there exist $a_{1}, a_{2}, a_{3} \in \mathbb{R}$ such that (1.2) has one non-trivial limit cycle.

With the change of variables $t \rightarrow \pi / 2-t$, one obtains similar results replacing $i$ by $j$, and interchanging the subindices 1 and 2 . 
Remark 3.4. The proof of the theorem consists of studying the stability near the origin and near infinity. The conditions $\left(n_{1}-n_{2}\right)\left(\frac{n_{3}}{k_{2}}-n_{2}\right)<0$ and $\left(n_{1}-n_{2}\right)\left(\frac{n_{1}}{k_{2}}-\frac{n_{2}}{k_{1}}+\frac{n_{3}}{k_{1} k_{2}}\right)<0$ will imply that the stabilities are opposite, and therefore there exists an odd number of non-trivial limit cycles. If one of the inequalities is reversed then there are an even number of non-trivial limit cycles or a semi-hyperbolic one, so that one can not conclude that there exists a limit cycle different from $x=0$.

The remaining conditions will imply that the stability near infinity is determined by what happens around $t=0$.

Proof. The proof will follow by computing the power series of $\Delta\left(x, a_{1}, a_{2}, a_{3}\right)$ in terms of $a_{1}, a_{2}$ around $a_{1}=a_{2}=0$.

$$
\begin{aligned}
& u\left(\pi, x, a_{1}, a_{2}, a_{3}\right)=u\left(\pi, x, 0,0, a_{3}\right)+u_{a_{1}}\left(\pi, x, 0,0, a_{3}\right) a_{1}+u_{a_{2}}\left(\pi, x, 0,0, a_{3}\right) a_{2} \\
& +u_{a_{1}^{2}}\left(\pi, x, 0,0, a_{3}\right) \frac{a_{1}^{2}}{2}+u_{a_{1} a_{2}}\left(\pi, x, 0,0, a_{3}\right) a_{1} a_{2} \\
& +u_{a_{2}^{2}}\left(\pi, x, 0,0, a_{3}\right) \frac{a_{2}^{2}}{2}+o\left(a_{1}^{2}, a_{1} a_{2}, a_{2}^{2}\right), \\
& u\left(\pi, x, a_{1},-a_{2}, a_{3}\right)=u\left(\pi, x, 0,0, a_{3}\right)+u_{a_{1}}\left(\pi, x, 0,0, a_{3}\right) a_{1}-u_{a_{2}}\left(\pi, x, 0,0, a_{3}\right) a_{2} \\
& \quad+u_{a_{1}^{2}}\left(\pi, x, 0,0, a_{3}\right) \frac{a_{1}^{2}}{2}-u_{a_{1} a_{2}}\left(\pi, x, 0,0, a_{3}\right) a_{1} a_{2} \\
& +u_{a_{2}^{2}}\left(\pi, x, 0,0, a_{3}\right) \frac{a_{2}^{2}}{2}+o\left(a_{1}^{2}, a_{1} a_{2}, a_{2}^{2}\right) .
\end{aligned}
$$

By Proposition 2.1,

$$
\begin{aligned}
\Delta\left(x, a_{1}, a_{2}, a_{3}\right) & =u\left(\pi, x, a_{1}, a_{2}, a_{3}\right)-u\left(\pi, x, a_{1},-a_{2}, a_{3}\right) \\
& =2 u_{a_{2}}\left(\pi, x, 0,0, a_{3}\right)+2 u_{a_{1}, a_{2}}\left(\pi, x, 0,0, a_{3}\right) a_{1} a_{2}+o\left(a_{1}^{2}, a_{1} a_{2}, a_{2}^{2}\right)
\end{aligned}
$$

Choosing $a_{1}, a_{2}$ small enough, and $a_{3}<0$, one knows from (3.10) that

$$
\operatorname{sign} \Delta\left(x, a_{1}, a_{2}, a_{3}\right)=a_{1} a_{2} \operatorname{sign}\left(n_{1}-n_{2}\right), \quad \text { for } x \text { small enough. }
$$

We shall prove that

$$
\begin{aligned}
\operatorname{sign} \Delta\left(x, a_{1}, a_{2}, a_{3}\right) & =a_{1} a_{2} \operatorname{sign} u_{a_{1} a_{2}}\left(\pi, x, 0,0, a_{3}\right) \\
& =a_{1} a_{2} \operatorname{sign}\left(n_{2}-n_{1}\right), \quad \text { for } x \text { large enough. }
\end{aligned}
$$

Therefore, there exists a limit cycle for $a_{1}, a_{2}$ small enough, and $a_{3}<0$.

We shall denote $u\left(t, x, 0,0, a_{3}\right)$ as $u(t)$ or simply as $u$. Differentiating with respect to $a_{1}$ in (1.2),

$$
u_{a_{1}}^{\prime}(t)=A_{1}(t) u^{n_{1}}(t)+n_{3} a_{3} A_{3}(t) u^{n_{3}-1}(t) u_{a_{1}}(t) .
$$


Since $u_{a_{1}}(0)=0$, integrating over $[0, \pi]$,

$$
\begin{aligned}
u_{a_{1}}(t) & =\int_{0}^{t} A_{1}(s) u^{n_{1}}(s) \exp \left(\int_{s}^{t} n_{3} a_{3} A_{3}(t) u^{n_{3}-1}(\tau) d \tau\right) d s \\
& =u^{n_{3}}(t) \int_{0}^{t} A_{1}(s) u^{n_{1}-n_{3}}(s) d s .
\end{aligned}
$$

Arguing analogously with $a_{2}$,

$$
u_{a_{2}}(t)=u^{n_{3}}(t) \int_{0}^{t} A_{2}(s) u^{n_{2}-n_{3}}(s) d s .
$$

Thus $u_{a_{2}}(\pi)=0$.

Differentiating with respect to $a_{1}$ and $a_{2}$ in (1.2) and evaluating at $a_{1}=$ $a_{2}=0$, one has

$$
\begin{aligned}
u_{a_{1} a_{2}}^{\prime}= & n_{1} A_{1} u^{n_{1}-1} u_{a_{2}}+n_{2} A_{2} u^{n_{2}-1} u_{a_{1}} \\
& +n_{3}\left(n_{3}-1\right) a_{3} A_{3} u^{n_{3}-2} u_{a_{1}} u_{a_{2}}+n_{3} a_{3} A_{3} u^{n_{3}-1} u_{a_{1} a_{2}} .
\end{aligned}
$$

Since $u(0)=0$, integrating over $[0, \pi]$,

$$
\begin{aligned}
u_{a_{1} a_{2}}(\pi)= & \int_{0}^{\pi}\left(n_{1} A_{1} u^{n_{1}-1} u_{a_{2}}+n_{2} A_{2} u^{n_{2}-1} u_{a_{1}}\right. \\
& \left.+n_{3}\left(n_{3}-1\right) a_{3} A_{3} u^{n_{3}-2} u_{a_{1}} u_{a_{2}}\right) e^{\int_{t}^{\pi} n_{3} a_{3} A_{3} u^{n_{3}-1} d s} d t .
\end{aligned}
$$

Since $x^{\prime}=a_{3} A_{3}(t) x^{n_{3}}$, one has

$$
\int_{s}^{t} n_{3} a_{3} A_{3}(\tau) u^{n_{3}-1}(\tau) d \tau=\int_{s}^{t} n_{3} \frac{u^{\prime}(\tau)}{u(\tau)} d \tau=\log \left(u^{n_{3}}(t) / u^{n_{3}}(s)\right) .
$$

Therefore,

$$
\begin{aligned}
u_{a_{1} a_{2}}(\pi)=u^{n_{3}}(\pi) \int_{0}^{\pi} & \left(n_{1} A_{1} u^{n_{1}-1-n_{3}} u_{a_{2}}+n_{2} A_{2} u^{n_{2}-1-n_{3}} u_{a_{1}}\right. \\
& \left.+n_{3}\left(n_{3}-1\right) a_{3} A_{3} u^{-2} u_{a_{1}} u_{a_{2}}\right) d t .
\end{aligned}
$$

Renaming the factors of the previous expression we get

$$
u_{a_{1} a_{2}}(\pi)=u^{n_{3}}(\pi)\left(S_{1}(x)+S_{2}(x)+S_{3}(x)\right),
$$

where

$$
\begin{aligned}
& S_{1}(x)=\int_{0}^{\pi} n_{1} A_{1}(s) u^{n_{1}-1}(s) F_{2}(s) d s, \\
& S_{2}(x)=\int_{0}^{\pi} n_{2} A_{2}(s) u^{n_{2}-1}(s) F_{1}(s) d s, \\
& S_{3}(x)=\int_{0}^{\pi} n_{3}\left(n_{3}-1\right) a_{3} A_{3}(s) u^{2 n_{3}-2}(s) F_{1}(s) F_{2}(s) d s, \\
& F_{k}(t)=\int_{0}^{t} A_{k}(s) u^{n_{k}-n_{3}}(s) d s, \quad k=1,2 .
\end{aligned}
$$


Now we shall use symmetries to simplify (3.12). Firstly, note that

$$
u(t, x)=\frac{x}{\left(1+a_{3}\left(1-n_{3}\right) x^{n_{3}-1} I_{A_{3}}(t)\right)^{\frac{1}{n_{3}-1}}}, \quad I_{A_{3}}(t)=\int_{0}^{t} A_{3}(s) d s .
$$

Thus, the function $t \rightarrow u(t-k \pi / 2)$ is even for every $k \in \mathbb{Z}$. If one chooses $a_{3}<0$ then $u(t, x)$ is defined for every $t$ for any $x$, it is decreasing in $(0, \pi / 2)$, and its maxima are at $k \pi$.

Since the function $s \rightarrow A_{2}(\pi / 2-s) u^{n_{2}-n_{3}}(\pi / 2-s)$ is odd, then $F_{2}(\pi / 2-t)$ is even and

Since

$$
S_{1}(x)=2 \int_{0}^{\pi / 2} n_{1} A_{1}(t) u^{n_{1}-1}(t) F_{2}(t) d t .
$$

$$
\int_{0}^{\pi} n_{2} A_{2} u^{n_{2}-1}(t) F_{1}(\pi / 2) d t=0
$$

then

$$
S_{2}(x)=\int_{0}^{\pi} n_{2} A_{2} u^{n_{2}-1}(t)\left(F_{1}(t)-F_{1}(\pi / 2)\right) d t .
$$

Now observe that

$$
t \rightarrow n_{2} A_{2} u^{n_{2}-1}(\pi / 2-t)\left(F_{1}(\pi / 2-t)-F_{1}(\pi / 2)\right)
$$

is an even function. Thus,

$$
S_{2}(x)=2 \int_{0}^{\pi / 2} n_{2} A_{2} u^{n_{2}-1}(t)\left(F_{1}(t)-F_{1}(\pi / 2)\right) d t .
$$

Analogously, as

$$
\int_{0}^{\pi} n_{3}\left(n_{3}-1\right) a_{3} A_{3} u^{2 n_{3}-2}(t) F_{1}(\pi / 2) F_{2}(t) d t=0
$$

then

$$
S_{3}(x)=\int_{0}^{\pi} n_{3}\left(n_{3}-1\right) a_{3} A_{3}(t) u^{2 n_{3}-2}(t)\left(F_{1}(t)-F_{1}(\pi / 2)\right) F_{2}(t) d t .
$$

Moreover the function

$$
t \rightarrow A_{3}(\pi / 2-t) u^{2 n_{3}-2}(\pi / 2-t)\left(F_{1}(\pi / 2-t)-F_{1}(\pi / 2)\right) F_{2}(\pi / 2-t)
$$

is even, and consequently

$$
S_{3}(x)=2 \int_{0}^{\pi / 2} n_{3}\left(n_{3}-1\right) a_{3} A_{3}(t) u^{2 n_{3}-2}(t)\left(F_{1}(t)-F_{1}(\pi / 2)\right) F_{2}(t) d t .
$$

Note that for each $t \in(0, \pi / 2], u(t, x)$ tends to $u(t, \infty)$ as $x \rightarrow \infty$, where $u(t, \infty)=\left(\left(1-n_{3}\right) a_{3} I_{A_{3}}(t)\right)^{\frac{-1}{n_{3}-1}}=\left(\left(1-n_{3}\right) a_{3} t^{i_{3}+1}\right)^{\frac{-1}{n_{3}-1}}+o\left(t^{\frac{-i_{3}-1}{n_{3}-1}}\right)$.

We shall write $S_{k}(\infty)$ to denote $S_{k}(x)$ when we replace $u(t, x)$ by $u(t, \infty)$. Since the convergence is monotonic, $S_{k}(x) \rightarrow S_{k}(\infty)$ as $x \rightarrow \infty, k=1,2,3$.

Consider the following two cases: 
$\left(C_{1}\right)$ Condition $(a)$ guarantees that $k_{1}>0$ and $k_{2}>0$. Thus, $F_{j}(t)$ is defined for every $t$ close to zero, $j=1,2$. From $n_{1}<n_{3}$, one obtains

$$
1+i_{1}-\frac{\left(n_{1}-1\right)\left(i_{3}+1\right)}{n_{3}-1}+k_{2}>0,
$$

which implies $S_{1}(\infty)<\infty$. Condition $(b)$ is equivalent to

$$
k^{-\stackrel{\text { def }}{=}} i_{2}-\left(n_{2}-1\right) \frac{\left(i_{3}+1\right)}{n_{3}-1} \leq-1 .
$$

Moreover

$$
S_{2}(\infty)+S_{3}(\infty)=\int_{0}^{\pi / 2}\left(K_{1}\left(\frac{n_{3}}{k_{2}}-n_{2}\right) t^{k^{-}}+o\left(t^{k^{-}}\right)\right) d t
$$

with

$$
K_{1}=2\left(\left(1-n_{3}\right) a_{3}\right)^{\frac{n_{2}-1}{1-n_{3}}} F_{1}(\pi / 2)>0 .
$$

Then $S_{2}(x)+S_{3}(x) \rightarrow S_{2}(\infty)+S_{3}(\infty)$ and

$$
\lim _{x \rightarrow \infty} u_{a_{1} a_{2}}(\pi, x)=\operatorname{sign}\left(\frac{n_{3}}{k_{2}}-n_{2}\right) \infty .
$$

$\left(C_{2}\right)$ Condition $(a)$ guarantees that $k_{1}<0$ and $k_{2}>0$. Condition $(b)$ is equivalent to

$$
k^{+} \stackrel{\text { def }}{=} 1+i_{1}+i_{2}-\left(n_{1}+n_{2}-n_{3}-1\right) \frac{\left(i_{3}+1\right)}{n_{3}-1} \leq-1
$$

Moreover

$$
S_{1}(\infty)+S_{2}(\infty)+S_{3}(\infty)=\int_{0}^{\pi / 2}\left(K_{2}\left(\frac{n_{1}}{k_{2}}-\frac{n_{2}}{k_{1}}+\frac{n_{3}}{k_{1} k_{2}}\right) t^{k^{+}}+o\left(t^{k^{+}}\right)\right) d t
$$

with

$$
K_{2}=2\left(\left(1-n_{3}\right) a_{3}\right)^{\frac{n_{1}+n_{2}-n_{3}-1}{1-n_{3}}}>0 .
$$

Then $S_{1}(x)+S_{2}(x)+S_{3}(x) \rightarrow S_{1}(\infty)+S_{2}(\infty)+S_{3}(\infty)$ and

$$
\lim _{x \rightarrow \infty} u_{a_{1} a_{2}}(\pi, x)=\operatorname{sign}\left(\frac{n_{1}}{k_{2}}-\frac{n_{2}}{k_{1}}+\frac{n_{3}}{k_{1} k_{2}}\right) \infty .
$$

In both cases, $u_{a_{1} a_{2}}(\pi, x)$ tends to $\pm \infty$ when $x \rightarrow \infty$, and $\operatorname{sign}\left(u_{a_{1} a_{2}}(\pi, x)\right)$ is opposite to the sign of $\left(n_{1}-n_{2}\right)$ by Condition $(c)$. Therefore we obtain a limit cycle by a Hopf bifurcation.

To prove the second part of the result, it is enough to perform the change of variable $t \rightarrow \pi / 2-t$ and apply the statement already proved.

Example 3.5. If $n_{1}<n_{2}<n_{3}$, then Theorem 3.2 does not apply while Theorem 3.3 does (with the convenient choice of $i_{1}, i_{2}, i_{3}$ ). In other words, Abelian integrals obtain limit cycles that Theorem 3.2 does not.

Indeed, since $\left(i_{3}+1\right)\left(n_{3}-n_{1}\right)<\left(i_{3}+1\right)\left(n_{3}-1\right)$, then Theorem 3.3 and Theorem 3.2 do not apply simultaneously. Taking for instance, $n_{1}=4$, $n_{2}=3, n_{3}=2, i_{1}=9, i_{2}=2, i_{3}=1$, one obtains an example for which Theorem 3.2 applies but Theorem 3.3 does not. 


\section{REFERENCES}

[1] A. Álvarez, J.L., Bravo, M. Fernández, The number of limit cycles for generalized Abel equations with periodic coefficients of definite sign, Commun. Pure Appl. Anal. 8-5, (2009), 1493-1501.

[2] M.J. Álvarez, J.L. Bravo, M. Fernández, Uniqueness of limit cycles for polynomial first-order differential equations, J. Math. Anal. Appl. 360-1, (2009), 168-189.

[3] M.J. Álvarez, J.L. Bravo, M. Fernández, Abel-like differential equations with a unique limit cycle, Nonlinear Analy. TMA, 74-11, (2011) 3694-3702.

[4] M.J. Álvarez, A. Gasull, H. Giacomini, A new uniqueness criterion for the number of periodic orbits of Abel equations, J. Differential Equations 234, (2007), 161-176.

[5] J.L. Bravo, M. Fernández, Limit cycles of non-autonomous scalar odes with two summands, Commun. Pure Appl. Anal., in press.

[6] J.L. Bravo, J. Torregrosa, Abel-like equations with no periodic solutions, J. Math. Anal. Appl., 342, (2008), 931-942.

[7] M. Calanchi, B. Ruf, On the number of closed solutions for polynomial ODE'S and a special case of Hilbert's 16th problem, Adv. Differential Equations 7(2), (2002), 197216.

[8] L.A. Cherkas, Number of limit cycles of an autonomous second-order system, Differentsial'nye Uravneniya 12, (1975), 944-946.

[9] J. Devlin, N.G. Lloyd, J.M. Pearson, Cubic systems and Abel equations, J. Differential Equations, 147, (1998), 435-454.

[10] A. Gasull and A. Guillamon. Limit cycles for generalized Abel equations, Int. J. Bif. Chaos, 16, (2006), 3737-3745.

[11] A. Gasull, J. Llibre, Limit cycles for a class of Abel equations, SIAM J. Math. Anal., 21-5 (1990), 1235-1244.

[12] A. Gasull, R. Prohens, J. Torregrosa, Limit cycles for rigid cubic systems, J. Math. Anal. Appl. 303, (2005), 391-404.

[13] A. Gasull, J. Torregrosa, Some results on rigid systems, In International Conference on Differential Equations (Equadiff-2003), World Sci. Publ., Hackensack, NJ. (2005), 340-345.

[14] J. Huang, Y. Zhao, Periodic solutions for equation $\dot{x}=A(t) x^{m}+B(t) x^{n}+C(t) x^{l}$ with $A(t)$ and $B(t)$ changing signs, J. Differential Equations, 253, (2012), 73-99.

[15] Y. Ilyashenko, Hilbert-type numbers for Abel equations, growth and zeros of holomorphic functions, Nonlinearity, 13, (2000), 1337-1342.

[16] H.G. Kaper, M.K. Kwong, Uniqueness for a class of nonlinear initial value problems, J. Math. Anal. Appl. 130 (1988) 467-473.

[17] A. Lins Neto, On the number of solutions of the equation $\frac{d x}{d t}=\sum_{j=0}^{n} a_{j}(t) x^{j}, 0 \leq$ $t \leq 1$, for which $x(0)=x(1)$, Inv. Math. 59, (1980), 67-76.

[18] V.A. Pliss, Non local problems of the theory of oscillations, Academic Press, New York, 1966.

Departament de Matemàtiques i Informàtica, Universitat de les Illes BaLears, 07122, Palma de Mallorca, Spain

E-mail address: chus.alvarez@uib.es

Departamento de Matematicas, Universidad de Extremadura, 06071 BadaJOZ, SPAIN

E-mail address: trinidad@unex.es

Departamento de Matematicas, Universidad de Extremadura, 06071 BadaJOZ, SPAIN

E-mail address: ghierro@unex.es 